\title{
EDITORIAL Sexual function in women with heart disease and coronary artery disease
}

International Journal of Impotence Research (2007) 19, 341-342; doi:10.1038/sj.ijir.3901543

Satisfying sexual activity requires adequate sexual function in both men and women. Sex life is considered as an essential aspect of quality of life, and distortions of former functionality can easily result in disastrous consequences, such as psychosocial conflicts, separation, depression and even increased morbidity and mortality. In 1970, Hellerstein and Friedman ${ }^{1}$ described the problems and risks of sexual function in the post-coronary patient, but the close connection between sexual dysfunction and organic diseases is not a new association in our times. The Austrian physician Wilhelm Reich, 1897-1957 a student of Freud, also worked on the theory of the sexual etiology of disease, resulting in the factual and verifiable discovery of Orgone energy (biophysical and cosmic energy). His research revealed that life abides by the orgonotic formula of 'tension, charge, discharge, relaxation'. Dysfunction is brought about through the blockage or cessation of this flow in the form of 'armor' (energy stricture) or 'anorgonia' (energy diffusion), which ultimately leads to disease (in his studies, to cancer), in contrast to 'full orgastic potency'. This orgonotic formula obviously represents the physiologic events of male erection and orgasm that allows the 'release of stress followed by pleasant conduct'. If disturbed, this ultimately results in organic pathology, mostly of chronic character. An acute event such as acute myocardial infarction, on the other hand, disrupts a 'normal physiologic state' that oftentimes results in dysfunctional sequelae, especially for hedonistic activities, which may require hemodynamic challenge. Conversely, studies on patients with heart failure as an example for a chronic but often compensated condition, demonstrated a higher than expected rate of sexual dysfunction, irrespective of reduced physical conditioning or capabilities. ${ }^{2}$

Scientific data in women are lacking, and it is unclear, why? Some explanations are: (1) sexual function is not as easily qualitatively or quantitatively measured in women compared to the obvious impaired stiffness of a man's genital and there is a lack of a well-defined and widely accepted classification system; (2) anecdotal reports reveal that sexual function seems not to play that overwhelming role for many women at higher ages compared with men; (3) the importance of sexual activity in women is often geared more towards a broader spectrum of sexual sensuality; (4) there is more general acceptance of cessation of sexual activity in middle-aged and older women that is only comparable to men at very high ages or in severe health conditions; (5) we (physicians) do not pay enough attention and a male physician might not feel comfortable in asking female patients about their sex life; (6) some women might not want to address this issue with a health care provider.

On the other hand, chronic diseases such as coronary artery disease (CAD) are associated with depressive symptoms that ultimately can result in worsened sexual function, either through depression itself or by antidepressive drugs. ${ }^{3}$ Side effects of several antidepressive agents include reduced libido in both women and men and erectile dysfunction. In several cases, however, it is not clear whether depression had caused sexual dysfunction or whether sexual dysfunction caused depression, but obviously both conditions unite in fortification.

Female sexual dysfunction oftentimes encompasses more than just one impaired condition. Also in women, sexual dysfunction is age-related, progressive and highly prevalent affecting $30-50 \%$ of American women. Based on the National Health and Social Life Survey of 1749 women, $43 \%$ have complaints of sexual dysfunction (even though older women were not included). ${ }^{4}$ Recent studies evaluating the impact of age and estrogen status on the female sexual response demonstrated that older women and menopausal women not receiving hormone replacement therapy had decreased genital blood flow compared to controls. There is evidence, however, that disease processes and risks that are associated with male erectile dysfunction are also associated with female sexual dysfunction, indicating a common pathophysiologic mechanism. These conditions include aging, hypertension, cigarette smoking, hyperlipoproteinemia and factors that affect the vascular system potentially leading to endothelial dysfunction and atherosclerosis, as well as pelvic surgeries. ${ }^{5,6}$

The Diagnostic and Statistical Manual of Psychiatry (DSM IV) defines female sexual dysfunction based on Masters' and Johnson's characterization of the female sexual response that consists of the four phases excitement, plateau, orgasmic and resolution phases $^{7}$ and on Kaplan's three-phase model consisting of desire, arousal and orgasm. ${ }^{8}$ The American 
Foundation of Urologic Disease (AFUD) Consensus Panel, however, had revised the terminology that now is based on four domains: libido, arousal, orgasm and satisfaction. ${ }^{9}$ Female sexual dysfunction is now often subdivided as lifelong versus acquired, generalized versus situational and organic versus psychogenic, or mixed and encompasses several distinct disorders: (1) hypoactive sexual desire disorder, (2) sexual aversion disorder, (3) sexual arousal disorder, (4) orgasmic disorder, (5) sexual pain disorders such as dyspareunia, vaginismus or others. ${ }^{6}$

The article in this issue by Dr Kaya and coworkers describes for the first time a direct comparison between women of normal health with women with known CAD with regard to self-assessed sexual dysfunction by use of the Female Sexual Function Index (FSFI). As suspected but not shown in its clarity before, even within the relative small number of only 20 female patients with CAD, 12 women reported symptoms of sexual dysfunction compared with just five out of 15 healthy female age-matched volunteers. Even though the data would be more convincing if groups consisting of higher and equal numbers had been compared, these preliminary data demonstrate an increased incidence of sexual dysfunction in women with CAD. Of note, there are no data provided on the time course of the disease or concomitant conditions, such as hypertension, hyperlipoproteinemia or undiagnosed depression. In contrast, diabetes, depression, uncontrolled hypertension and recent smoking were all exclusion criteria. One might wonder which unusual selection of women was included that lacked almost all common risk factors but still had $C A D$. Moreover, chronic medication use that additionally might contribute to the development of sexual dysfunction has not been analyzed in the current study. Data on the kind of marital or extramartial (or neither or eventually both) sexual relationships or on the sexual partners are not given in detail hat could help to understand the reduced numbers of intercourse in women with CAD compared with controls. Neither therapeutical success nor numbers of stenoses of involved coronaries or the duration of the disease or its symptoms are correlated to the female sexual dysfunction index (FSDI) score. Whether pre- or peri- or post-menopausal status plays a role in the prevalence of FSD remains unknown from this small cohort. Even though there seems to be a difference in estradiol levels (with lower levels in women with CAD compared with controls), this difference did not reach significance in the present study but might lead to speculate a tendency towards reduced hormone levels in women with CAD.

Despite these critics, the presented data are important but might raise more questions. One evolving question is: can (successful) treatment of anginal symptoms (by either medication or revascularization procedures) improve sexual dysfunction in women? If female sexual dysfunction is thought be mainly related to endothelial dysfunction and the occurrence of FSD is more an epiphenomenon than a consequence of CAD, then successful treatment of a single coronary artery stenosis would not be expected to alleviate sexual dysfunction. If, on the other hand, FSD appears to be a natural consequence of clinically manifest CAD, therapy by use of vasocactive drugs or revascularization procedures that eliminate symptoms hypothetically could improve FSD. Lastly, the presented data from one distinct socio-cultural part of the world (Turkey) does not necessarily represent different settings in other continents, but cross-national/cross sociocultural comparisons are lacking. Despite several uncertainties and lack of data, however, Dr Kaya's paper shows a higher prevalence of FSD among women with CAD compared to healthy women and thus, contributes to the understanding of the importance of recognizing the potential impact of sexual dysfunction on quality of life in women with cardiovascular disorders. More data on female sexual disorder, in particular if associated with chronic diseases, hopefully will help to overcome our current negligence in addressing and studying this emotionally distressing, socially disruptive and pathophysiologically uncertain territory.

Disclosures: None.

\section{Ernst R Schwarz ${ }^{1,2}$ \\ ${ }^{1}$ Division of Cardiology \& Comprehensive} Transplant Center, Cedars Sinai Medical Center, Los Angeles, CA, USA and ${ }^{2}$ David Geffen School of Medicine, University of California Los Angeles (UCLA), Los Angeles, CA, USA E-mail: schwarze@cshs.org

\section{References}

1 Hellerstein HK, Friedman EH. Sexual activity and the postcoronary. Arch Intern Med 1970; 125: 987-999.

2 Schwarz ER, Rastogi S, Kapur V, Sulemanjee N, Rodriguez JJ. Erectile dysfunction in heart failure patients. J Am Coll Cardiol 2006; 19: 1111-1119.

3 Schwarz ER. Sex and the Heart. Friedel \& Ernst Academic Press: Los Angeles, 2006, (http://sexandtheheart.com).

4 Laumann EO, Paik A, Rosen RC. Sexual dysfunction in the United States: prevalence and predictors. JAMA 1999; 281: 537-544.

5 Berman JR, Berman L, Goldstein I. Female sexual dysfunction: incidence, pathophysiology, evaluation, and treatment options. Urology 1999; 54: 385-391.

6 Berman JR. Physiology of female sexual function and dysfunction. Int J Impot Res 2005; 17(Suppl 1): S44-S51.

7 Masters EH, Johnson VE. Human Sexual Response. Little Brown \& Co.: Boston, 1966.

8 Kaplan HS. The New Sex Therapy. Bailliere Tindall: London, 1974.

9 Basson R, Berman JR, Burnett A, Derogatis L, Ferguson D, Fourcroy J et al. Report on the international consensus development conference of female sexual dysfunction: definitions and classifications. J Urol 2000; 163: 888-893. 